\title{
Acknowledgement to Reviewers of Molbank in 2017
}

\author{
Molbank Editorial Office \\ MDPI AG, St. Alban-Anlage 66, 4052 Basel, Switzerland; molbank@mdpi.com
}

Published: 9 January 2018

Peer review is an essential part in the publication process, ensuring that Molbank maintains high quality standards for its published papers. In 2017, a total of 46 papers were published in the journal. Thanks to the cooperation of our reviewers, the median time to first decision was 12.5 days and the median time to publication was 26.5 days. The editors would like to express their sincere gratitude to the following reviewers for their time and dedication in 2017:

\begin{tabular}{|c|c|c|}
\hline Andrea, Penoni & Gray, Christopher A. & Nurieva, Evgeniya \\
\hline Ata, Athar & Grée, René & O'Donoghue, Annmarie \\
\hline Aubert, Emmanuel & Guazzelli, Lorenzo & Ogawa, Akiya \\
\hline Baek, Nam-In & Hatae, Noriyuki & Oka, Natsuhisa \\
\hline Bassin, Paul & Hawkins, Bill & Ostrowski, Stanisław \\
\hline Basso, Andrea & Heber, Dieter & Pastor, Isidro M. \\
\hline Berna, Jose & Henderson, William & Petrova, Krasimira T. \\
\hline Bianco, Armandodoriano & Hideg, Kálmán & Piccialli, Vincenzo \\
\hline Black, David & Hoz, Antonio De La & Poddel'sky, Andrey \\
\hline Boeré, René & Huang, Jiann-Jyh & R. Odell, Luke \\
\hline Boga, Carla & Husson, Jérôme & Reber, Christian \\
\hline Bruce, Alice & Inokuchi, Tsutomu & Ritch, Jamie \\
\hline Bunce, Richard A. & Ishikawa, Yoshinobu & Rodrigues, Catarina \\
\hline Castro, Maria & J Edagwa, Benson & Romieu, Anthony \\
\hline Centore, Roberto & Jenkins, Ian & Ryu, Shi Yong \\
\hline Chao, Chih-Hua & Kashman, Yoel & Sansano, Jose M. \\
\hline Chen, Jhy-Der & Katagiri, Hiroshi & Sarkar, Dhruba \\
\hline Cho, Chan Sik & Kim, Sung-Gon & Serra, Stefano \\
\hline Chu, Yen-ho & Kinthada, Ramakumar & Shaw, Subrata \\
\hline Chuang, Che-Ping & Kitano, Yoshikazu & Snyder, Nicole \\
\hline Costa, Susana P.G. & Kitazawa, Takafumi & Speed, Alexander \\
\hline Curtis, Neil F. & Konno, Hiroyuki & Stanovnik, Branko \\
\hline Dasari, Ramesh & Kosmrlj, Janez & Stenstrøm, Yngve \\
\hline Dawe, Louise & Kurata, Hiroyuki & Stephen, Michael Rajesh \\
\hline Della Ca', Nicola & Lehnherr, Dan & Sugai, Takeshi \\
\hline Detert, Heiner & Litvić, Mladen & Tanaka, Fujie \\
\hline Diaba, Faïza & Lucas, Nigel T. & Tanaka, Shinji \\
\hline Dibble, Peter & Luconi, Lapo & Thierry, Ollevier \\
\hline Dolzhenko, Anton V. & Macchi, Beatrice & Thomas, Jim \\
\hline Fattuoni, Claudia & Manos, Manolis & Tiefenbacher, Konrad \\
\hline Ferretti, Valeria & Massa, Antonio & Torroba, Tomas \\
\hline Fraile, Alberto & Mieczkowski, Adam & Tranmer, Geoffrey \\
\hline Frongia, Angelo & Monclus, Michel & Trivedi, Evan R. \\
\hline Gerken, Michael & Nemec, Ivan & Tsubaki, Kazunori \\
\hline Gordon, Keith & Nemoto, Tetsuhiro & Uivarosi, Valentina \\
\hline Gotsbacher, Michael & Novak, Lajos & Van Hecke, Kristof \\
\hline
\end{tabular}


Vila, Carlos

Walton, John

White, Robert L.

Winne, Johan M.

Wujec, Monika

\author{
Yang, Xiu wei \\ Yerabolu, Jayasudhan Reddy \\ Yin, Sheng \\ Ylijoki, Kai E. O. \\ Yokomatsu, Tsutomu
}

Zaharia, Valentin

Zakrzewski, Jerzy

Žukauskaitė, Asta

(c) 2018 by the authors; licensee MDPI, Basel, Switzerland. This article is an open access article distributed under the terms and conditions of the Creative Commons Attribution (CC-BY) license (http://creativecommons.org/licenses/by/4.0/). 